\title{
Leçons tirées des tragédies liées aux préparations magistrales de médicaments
}

\author{
par Eric S Kastango
}

$\mathrm{P}_{\mathrm{t}}^{\mathrm{h}}$ usieurs mois se sont écoulés depuis la terrible nouvelle de la tragédie secondaire à la préparation magistrale d'un médicament à la pharmacie New England Compounding Center (NECC) de Framingham, au Massachusetts. Une éclosion de méningite fongique à l'automne 2012 a été associée à des injections d'acétate de méthylprednisolone contaminé préparé par le NECC. Selon les Centers for Disease Control and Prevention (CDC) des États-Unis, ${ }^{1}$ en date du 11 mai 2013, on dénombrait 55 décès et un total de 741 cas d'infections attribuables à la contamination, et ces nombres ne cessent de croître. Les patients qui ont reçu des médicaments de cette pharmacie ont également été victimes d'infections paramédullaires, rachidiennes ou articulaires périphériques.

L'Institute for Safe Medication Practices (ISMP) des ÉtatsUnis compile une liste choisie d'accidents associés à des préparations magistrales depuis les années 1990. ${ }^{2}$ Pour des raisons d'ordre juridique, de nombreux accidents associés à des préparations magistrales ne sont jamais divulgués au public, mais la Food and Drug Administration (FDA) des États-Unis inspecte activement les pharmacies spécialisées dans les préparations magistrales et que l'on soupçonne ou que l'on sait avoir des problèmes à grande échelle concernant la qualité ou les préparations magistrales. ${ }^{3,4}$

Les problèmes touchant les préparations magistrales ne sont pas cantonnés qu'aux États-Unis. Récemment, plus de 1200 patients atteints de cancer en Ontario et au NouveauBrunswick ont reçu des solutions chimiothérapeutiques à des concentrations inférieures aux valeurs précisées sur les étiquettes. Il semble que les questions de champs de compétence ayant trait à la supervision adéquate de cette opération et autres soient similaires aux problèmes cernés dans le cadre des événements touchant le NECC. 5,6

Il est temps de regarder en face notre naïveté et notre arrogance occasionnelle à propos des pratiques sécuritaires de préparation magistrale des médicaments. Nous devons tirer des leçons de ces événements et prendre les mesures nécessaires pour nous assurer de faire tout ce qui est possible pour protéger les patients et réduire le risque de préparations magistrales stériles inadéquates. Personnellement, j'ai été profondément touché par les événements du NECC et je suis toujours perplexe quant aux façons de faire de certains de mes collègues qui continuent pourtant de croire que les principes de contrôle de la contamination et les systèmes qualité robustes ne sont pas pour eux. En tant que pharmacien et potentiellement futur patient, je vous demande : "Que faites-vous pour vos patients actuels et futurs à la lumière de ces événements récents? "

Que vous soyez d'accord ou non avec le chapitre <797> de la United States Pharmacopeia (USP) et d'autres chapitres de la USP essentiels à la préparation magistrale des médicaments ${ }^{7}$ ou avec le guide de préparation magistrale du Pharmaceutical Inspection Cooperation Scheme, ${ }^{8}$ le message est on ne peut plus clair. Le statu quo n'est plus acceptable. Nous devons adopter les normes et les lignes directrices fondées sur des données scientifiques probantes et les meilleures pratiques qui ont été soigneusement considérées par le truchement de commentaires du public et d'une série d'examens.

Plus particulièrement, le temps est venu de réaliser des évaluations du risque des méthodes de préparation magistrale utilisées dans nos propres pharmacies, de sorte que les risques inhérents puissent être cernés et réduits au moyen de politiques et procédures détaillées, de programmes de formation rigoureux et de systèmes qualité. Les employés de la pharmacie doivent connaître non seulement les attentes à leur endroit lorsqu'ils préparent des formes pharmaceutiques stériles, mais la raison de ces attentes. Nous devons nous éloigner du modèle pur de formation en apprentissage au profit de méthodes et de procédures qui établissent des bases solides pour l'exécution d'un travail de qualité qui évitera les erreurs de préparation magistrale.

Les praticiens en pharmacie sont de plus en plus sollicités pour préparer des formes pharmaceutiques magistrales stériles non disponibles dans le commerce, utilisant parfois des ingrédients pharmaceutiques actifs non stériles. La manipulation et la transformation de ces composés en formes 
pharmaceutiques stériles adéquates peuvent nécessiter une expérience et une expertise supérieures aux compétences du personnel d'une pharmacie donnée. Dans de tels cas, nous devons tenir compte de ces limites et reconnaître les risques qu'elles constituent pour nos patients. Plus particulièrement, seules des procédures rigoureuses et validées doivent être utilisées. Nous avons la responsabilité de voir à ce que les préparations magistrales de médicaments correspondent aux produits attendus et, dans la mesure du possible, d'en assurer et d'en vérifier la stérilité. De la même façon, si nous n'avons pas l'expertise requise, nous ne devons pas effectuer ces tâches.

Historiquement, les pharmaciens préparaient les médicaments de façon "juste-à-temps ». Cependant, les modèles de distribution actuels font en sorte quil faut entreposer une plus grande quantité de préparations magistrales stériles à la température ambiante, ce qui incite ainsi à repousser les dates limites d'utilisation. Les tests de stérilité décrits dans le chapitre $<71>$ de la USP sont des tests de qualité industrielle conçus pour déceler les cas de contamination massive, mais ils sont difficiles à réaliser sur de petits lots de médicaments pour lesquels les dates limites d'utilisation désirées dépassent celles décrites dans le chapitre < 797> de la USP. Par conséquent, le fait de passer le test du chapitre $<71>$ de la USP est un faux indicateur de qualité. Nous devons nous attarder à incorporer l'élément qualité dans toutes les préparations magistrales stériles en mettant au point des processus rigoureux et éprouvés qui permettent d'obtenir des médicaments de la qualité attendue (en termes de concentration et de stérilité).

Lorsque les armées de Jules César ont traversé le Rubicon en 49 avant J.-C., il a dit " alea jacta est » — « Le sort en est jeté ». La pharmacie a traversé son propre Rubicon. Les inspections des pharmacies spécialisées dans les préparations magistrales aux États-Unis par la FDA ont été musclées et je salue les mesures prises pour évaluer l'ampleur de certaines grandes « pharmacies non traditionnelles ». Plusieurs organismes de réglementation de la pharmacie d'États américains effectuent aujourd'hui des inspections ou ont recours à d'autres méthodes pour évaluer la conformité opérationnelle au chapitre <797> de la USP des pharmacies communautaires et des pharmacies d'hôpital (peu importe si les lois de l'État exigent ou non une telle conformité). Certains États ont renforcé leurs règlements sur les préparations magistrales stériles, alors que des dizaines d'autres se mobilisent pour corriger les lacunes de leur législation. La National Association of Boards of Pharmacy s'est associée à des spécialistes en préparation magistrale de médicaments pour proposer de l'aide à la formation sur les préparations magistrales stériles aux inspecteurs des organismes de réglementation de la pharmacie des États. Le CDC appuie le chapitre <797> de la USP et a publié des lignes directrices sur les pratiques d'injection sécuritaires afin de prévenir la transmission d'infections aux patients.' Les lignes directrices de la Société canadienne des pharmaciens d'hôpitaux (SCPH) sur la préparation des produits stériles $<10>$ sont présentement en révision et les lignes révisées tiendront très probablement compte des leçons tirées de ces événements et d'autres situations.

Les données scientifiques sur lesquelles s'appuient les exigences du chapitre $<797>$ de la USP et les lignes directrices de la SCPH sur la préparation des produits stériles ne sont pas nouvelles. Lorsque l'American Society of Hospital Pharmacists (maintenant l'American Society of Health-System Pharmacists) a publié ses lignes directrices en 1993, Kenneth E. Avis, vu par beaucoup comme le père des bonnes pratiques de préparation magistrale en pharmacie, aurait déclaré : "Les données scientifiques ne sont pas nouvelles, mais sont plutôt de vieilles nouvelles que peu se donnaient la peine de tenir compte. " Comme l'ISMP l'a dit, " Malheureusement, il y a trop de gens du monde de la santé qui croient que, parce que ça ne leur est pas arrivé, les mauvaises expériences des autres ne les concernent pas." Alors, rendons hommage aux victimes en faisant les choses correctement dès maintenant.

\section{[Traduction par l'éditeur]}

\section{Références}

1. Multi-state meningitis outbreak-current case count. Persons with fungal infections linked to steroid injections, by state [plan]. Atlanta (GA) : Centers for Disease Control and Prevention; 6 mai 2013. Publié au www.cdc.gov/ hai/outbreaks/meningitis-map-large.html. Consulté le 11 mai 2013.

2. Sterile compounding tragedy is a symptom of a broken system on many levels. ISMP Med Saf Alert, 18 octobre 2012. Publié au www.ismp.org/ Newsletters/acutecare/showarticle.asp?id=34. Consulté le 11 mai 2013.

3. 2013 pharmacy inspections. Silver Spring (MD) : US Department of Health and Human Services, Food and Drug Administration; 2013. Publié au www.fda.gov/AboutFDA/CentersOffices/OfficeofGlobalRegulatory OperationsandPolicy/ORA/ORAElectronicReadingRoom/ucm340853. htm. Consulté le 11 avril 2013.

4. [Rapport d'inspection]. Silver Spring (MD) : US Department of Health and Human Services, Food and Drug Administration; 2012. Publié au www.fda.gov/downloads/AboutFDA/CentersOffices/OfficeofGlobal RegulatoryOperationsandPolicy/ORA/ORAElectronicReadingRoom/ UCM325980.pdf. Consulté le 11 avril 2013.

5. Zafar A. Chemotherapy outsourcing done by hospitals across Canada. CBC News, Health; 25 avril 2013. Publié au www.cbc.ca/news/health/ story/2013/04/24/chemotherapy-baxter.html. Consulté le 11 mai 2013.

6. Chemo scandal: Health Canada cracks down on private drug suppliers. TheSpec.com; 20 avril 2013. Publié au www.thespec.com/news/local/ article/922024--chemo-scandal-health-canada-cracks-down-on-privatedrug-suppliers. Consulté le 11 mai 2013.

7. USP [United States Pharmacopeia] 36-NF [National Formulary] 36. Rockville (MD) : US Pharmacopeial Convention; 2012.

8. PIC/S guide to good practices for the preparation of medicinal products in healthcare establishments. PE 010-3. Genève (Suisse) : Pharmaceutical Inspection Convention Secretariat and Pharmaceutical Inspection CoOperation Scheme (PIC/S); $1^{\text {er }}$ octobre 2008. Publié au www.picscheme.org/ pdf/23_pe0103-revisedgppguide.pdf. Consulté le 14 mai 2013.

9. Protect patients against preventable harm from improper use of singledose/single-use vials. Atlanta (GA) : Centers for Disease Control and Prevention, National Center for Emerging and Zoonotic Infectious Diseases, Division of Healthcare Quality Promotion; 2 mai 2012. Publié au www.cdc.gov/injectionsafety/CDCposition-SingleUseVial.html. Consulté le 11 avril 2013.

10. Lignes directrices sur la préparation des produits stériles dans les pharmacies. Ottawa (ON) : Société canadienne des pharmaciens d'hôpitaux; 1996. En révision. 
Eric S Kastango, MBA, RPh, FASHP, is President and CEO of ClinicallQ, LLC, and CriticalPoint, LLC, in Florham Park, New Jersey.

Divulgation d'intérêts concurrents : Eric Kastango est membre du personnel enseignant du Sterile Compounding Boot Camp présenté par CriticalPoint, LLC. II a agi comme témoin expert sur de nombreux sujets hors du cadre de cet éditorial pour le compte du Virginia Board of Pharmacy (États-Unis) et a reçu des honoraires comme conférencier et le remboursement de frais de déplacement et de réunion de nombreuses associations professionnelles de pharmacie. II a aussi touché des redevances de Pharmacy OneSource (Wolters Kluwer Health). Les opinions, les perceptions, les commentaires et les points de vue exprimés dans les présentes ou de toute autre manière sont uniquement ceux de l'auteur et ne doivent en aucun cas être interprétés comme la position officielle de tout organisme ou de toute personne vivante ou décédée.

\section{Adresse de correspondance :}

Eric S Kastango

Clinical IQ, LLC

235 Main Street, Suite 292

Madison NJ 07940 USA

Courriel : eric.kastango@clinicaliq.com
Cette collaboration spéciale s'inscrit dans une série continue d'éditoriaux rédigés par des pharmaciens très en vue, invités à commenter une question d'actualité pour la profession. Dans son choix d'un éditorialiste invité à commenter les enjeux et les préoccupations liés à la préparation magistrale des médicaments, le comité de rédaction voulait le point de vue d'un pharmacien exerçant à l'extérieur du Canada, en partie afin de souligner que les problèmes en question vont au-delà des frontières.

\title{
$\neg$ Canadian Society of Hospital Pharmacists Société canadienne des pharmaciens d'hôpitaux
}

\author{
Membership Year 2013/2014 \\ (July 1, 2013 - June 30, 2014)
}

Are you a member of the Canadian Society of Hospital Pharmacists?

CSHP has celebrated 66 years of inspiration and leadership among a rapidly growing network of over 3000 pharmacy colleagues. As a member of CSHP, you will have access to resources and opportunities that will enhance your career and help impact the direction of healthcare in Canada. Your participation in CSHP 2015, an initiative challenging all pharmacists to improve patient medication outcomes and safety through advancing pharmacy practice excellence by the year 2015, is a prime example.

We invite you to reap the benefits of an affordable membership with CSHP. For more information, please visit www.cshp.ca.

Membership Enquiries: Please contact Membership Services

Tel.: (613) 736-9733 Ext. 222 Fax: (613) 736-5660 Email: membershipservices@cshp.ca 